\title{
Viewpoint \\ The evolving paradigm of tissue-specific metastasis
}

Theresa A DiMeo ${ }^{1}$ and Charlotte Kuperwasser ${ }^{1,2}$

\author{
1Department of Anatomy and Cellular Biology, Tufts University School of Medicine, Boston, Massachusetts, USA \\ 2Department of Radiation Oncology, Tufts-New England Medical Center, Molecular Oncology Research Institute, Boston, Massachusetts, USA
}

Corresponding author: Charlotte Kuperwasser, charlotte.kuperwasser@tufts.edu

Published: 22 December 2005

This article is online at http://breast-cancer-research.com/content/8/1/301

(c) 2005 BioMed Central Ltd

\section{Introduction}

Breast cancer frequently metastasizes to bone, lung, liver, and brain, which is the major cause of morbidity and mortality associated with the disease [1]. The propensity with which breast cancer associates with certain tissue types in advanced stages of malignancy suggests a molecular basis for this attraction, but the mechanism remains unknown.

Previous dogma suggested a genetic basis for breast cancer metastasis as an extension of the stepwise transformation model, whereby the accumulation of genetic mutations confers selective survival and proliferative advantages to reach a malignant state of growth. Indeed, it was thought that several additional genetic alterations after initial transformation were required to confer metastatic ability [2]. Recent evidence has challenged this notion by suggesting that the genetic events necessary for primary tumor growth may also mediate metastatic behavior [3]. However, it is difficult to reconcile how the same genetic alterations could confer growth both in the breast microenvironment and in other tissue microenvironments.

Recent work has begun to shed some light on the molecular mechanisms that mediate tissue-specific metastasis and reconcile this conceptual conflict. However, whether the genes identified in these studies will elucidate the genetic and, hopefully, predicative underlying mechanism for metastasis, or rather represents an artifact of the model systems employed for gene candidate discovery remains uncertain.

\section{Changing paradigms in breast cancer metastasis}

The widely accepted hypothesis first reported by Paget suggests that breast cancer cells must express and utilize specific migratory and adhesion molecules that cross-react with those expressed in distant tissues in order to intravasate, survive, and proliferate in that microenvironment. However, when combined with the accepted mutation hypothesis, it is
Breast Cancer Research 2006, 8:301 (doi:10.1186/bcr1373)

difficult to conceive how breast cancer cells could be selected during the process of primary tumor growth in the breast stromal microenvironment also to express factors specific for other tissue microenvironments.

There is an alternative explanation, which has received less attention owing to the difficulty of studying the process. It suggests that once cells extravasate out of the primary tissue they genetically/epigenetically express migratory and adhesion molecules as a consequence of genetic instability and selection, which in turn facilitates their adhesion and survival in tissues that express the reciprocal growth factor or adhesion molecule [4].

However, recent studies have suggested a third possibility, that single cells within a heterogeneous population already express tissue-specific factors that would enable them to grow preferentially in the lung, bone, or adrenal microenvironments [5-7]. Although this work is intriguing and demonstrates a novel mechanism of tissue-specific metastasis, the model system utilized in those studies may not reflect human breast cancer metastasis, or even tissuespecific metastasis.

\section{Model systems of human breast cancer metastasis}

Model systems for the study of human breast cancer metastasis are currently limited. Several strategies have been developed to study metastasis from the mouse mammary gland. One involves the utilization of a sub-line of the invasive human breast cancer cell line MDA-MB-231, which was selected by serial in vivo passages for its ability to develop bone metastases when injected through the tail vein of immunocompromised mice [7]. This line, termed MDA-MB231Bo, along with MDA-MB-435 were the only two human breast cancer cell lines utilized to model and unravel the molecular mechanisms by which breast cancer metastasizes from the orthotopic site. Because the parental MDA-MB-231 cell line was isolated from a pleural effusion and is not 
metastatic from the mammary gland, and the MDA-MB-435 cell line exhibits a melanoma rather than a breast cancer phenotype, these cell lines may not accurately recapitulate breast cancer metastasis and so caution is required when translating the findings of such studies to humans.

Despite the caveats in the MDA-MB-231 cell line, this model was employed to isolate clones that metastasize to the lung and bone following injection of cells into the tail vein and left cardiac ventricle of mice, respectively [5-7]. These studies demonstrated the inherent variability in metastatic behavior and gene expression within the MDA-MB-231 parental cell line, and thus it was concluded that primary breast tumors are also composed of a heterogeneous population of cells with varying gene expression profiles and different metastatic capabilities.

The method of injection of these breast cancer cell lines may bias the metastatic phenotype. For example, cells injected through the tail vein will follow the venous flow and immediately encounter the lung capillary bed environment. Similarly, cells injected through the left heart ventricle will follow the arterial flow and deposit in organs encountered along this blood flow route (e.g. bone). Because this model system does not accurately reflect the metastatic process, does this work reflect human breast cancer metastasis, or even tissue-specific metastasis?

\section{Possible genes that mediate metastasis}

Given the clonal variability in breast cancer cells, microarray analysis was performed on tissue-specific clonal lines to examine what genes might mediate tissue selectivity during metastasis. Surprisingly, the various clones isolated from the MDA-MB-231 cell line exhibited distinct and differential in vitro transcriptional profiles, suggesting a genetic/epigenetic basis for the tissue-specific growth patterns of breast cancer. Although some biologically interesting and novel genes were identified by this process (e.g. ID1), several genes already implicated in tissue-specific metastasis were identified (e.g. MMP1, IL11, CXCR4, OPN), thus shedding no light on possible new candidates or mechanisms of action. Moreover, the experimental procedure raises doubts regarding how relevant these genes are in tissue-specific metastasis because several of them are known to mediate metastasis in a general manner and not necessarily specific to bone or lung (CXCR4, MMP1) [8,9].

Concomitant overexpression of several of the parental population genes identified by this approach conferred tissue-specific growth, highlighting the fact that no one gene was responsible for tissue-specific metastasis. Most importantly, when utilizing small interfering RNA technology to inhibit the expression of these genes in the clones, tissuespecific metastasis was inhibited but not primary tumor growth. This again suggested that a few critical genes could confer this process. Based on these results, a specific gene signature was obtained from several of these clones, and this list of genes could be used to identify whether a patientderived primary tumor will metastasize to the lung. It was surprising, however, that this approach was unable to predict whether a breast cancer could metastasize to bone, given that the majority of breast cancers metastasize to the skeleton. It may be the case that although breast cancer metastasis to bone may be inherent to all poor prognosis breast cancers [3], lung metastasis might require an even more defined molecular mechanism, which can be more readily identified by microarray analysis.

\section{The role of stroma in metastatic colonization}

Although inhibiting specific genes may prove to be important for preventing tissue-specific metastasis, the contribution of stroma to tumor growth should not be ignored. Tissue stroma has the ability to promote tumor growth [10]. For example, the bone microenvironment is highly dynamic, and this in turn is responsible for initiation and support of tumor growth. Application of bisphosphonates, which suppress bone turnover, can inhibit bone metastasis [11]. The importance of stroma has also been demonstrated on the expression pattern of MDA-MB-231 cells grown in vivo. The enzyme activity profile of these cells grown in culture compared with the same cells injected into the mammary fat pad of mice revealed profound differences, indicating that there was a stromal contribution to those grown in vivo [12]. The gene expression of cells cultured in the absence of tissue stroma could potentially change because of a loss of paracrine signaling. Based on this, it does not seem possible to identify accurately tissue-specific genes from microarray analysis performed on cells grown in culture, as was previously done [5-7]. Therefore, there remains much to be learned from future experiments conducted to identify markers and targets for metastasis, which take into account the contribution of the microenvironment.

\section{Conclusion}

The ability to predict and prevent metastasis based on the gene expression profile of a primary tumor would permit targeted therapeutic intervention and increase patient survival. However, the current models utilized to study breast cancer metastasis leave questions unanswered and are unable to identify accurately tissue-specific metastasis genes. In the future, it will be helpful to develop more accurate models that recapitulate breast cancer metastasis from an orthotopic site, and employ these systems to identify and elucidate the molecular and genetic mechanism that mediate this complex process.

\section{Competing interests}

The author(s) declare that they have no competing interests.

\section{Acknowledgements}

We would like to thank the National Institute of Health T32 HD007403-13 (TAD), and the Susan Komen and RB Sackler Foundations (CK). 


\section{References}

1. Weigelt B, Peterse JL, van 't Veel LJ: Breast cancer metastasis: markers and models. Nat Rev Cancer 2005, 5:591-602.

2. Fearon ER, Vogelstein B: A genetic model for colorectal tumorigenesis. Cell 1990, 61:759-767.

3. Bernards R, Weinberg RA: A progression puzzle. Nature 2002, 418:823.

4. Schardt JA, Meyer M, Hartmann $\mathrm{CH}$, Schubert $F$, Schmidt-Kittler O, Fuhrmann C, Polzer B, Petronio M, Eils R, Klein CA: Genomic analysis of single cytokeratin-positive cells from bone marrow reveals early mutational events in breast cancer. Cancer Cell 2005, 8:227-239.

5. Kang Y, Siegel PM, Shu W, Drobnjak M, Kakonen SM, CordonCardo C, Guise TA, Massague J: A multigenic program mediating breast cancer metastasis to bone. Cancer Cell 2003, 3: 537-549.

6. Minn AJ, Gupta GP, Siegel PM, Bos PD, Shu W, Giri DD, Viale A Olshen AB, Gerald WL, Massague J: Genes that mediate breast cancer metastasis to lung. Nature 2005, 436:518-524.

7. Minn AJ, Kang Y, Serganova I, Gupta GP, Giri DD, Doubrovin M, Ponomarev V, Gerald WL, Blasberg R, Massague J: Distinct organ-specific metastatic potential of individual breast cancer cells and primary tumors. J Clin Invest 2005, 115:44-55.

8. Muller A, Homey B, Soto H, Ge N, Catron D, Buchanan ME, McClanahan T, Murphy E, Yuan W, Wagner SN, et al:: Involvement of chemokine receptors in breast cancer metastasis. Nature 2001, 410:50-56.

9. Murray GI, Duncan ME, O'Neil P, McKay JA, Melvin WT, Fothergill JE: Matrix metalloproteinase-1 is associated with poor prognosis in oesophageal cancer. J Pathol 1998, 185:256-261.

10. Proia DA, Kuperwasser C: Stroma: tumor agonist or antagonist. Cell Cycle 2005, 4:1022-1025.

11. van der Pluijm G, Que I, Sijmons B, Buijs JT, Lowik CW, Wetterwald A, Thalmann GN, Papapoulos SE, Cecchini MG: Interference with the microenvironmental support impairs the de novo formation of bone metastases in vivo. Cancer Res 2005, 65:7682-7690.

12. Jessani N, Humphrey M, McDonald WH, Niessen S, Masuda K, Gangadharan B, Yates JR III, Mueller BM, Cravatt BF: Carcinoma and stromal enzyme activity profiles associated with breast tumor growth in vivo. Proc Natl Acad Sci USA 2004, 101: 13756-13761. 